Check for updates

Cite this: Phys. Chem. Chem. Phys., 2018, 20, 29061

Received 2nd October 2018, Accepted 5th November 2018 DOI: $10.1039 / \mathrm{c} 8 \mathrm{cp} 06161 \mathrm{k}$

rsc.li/pccp

\section{Vibrationally induced metallisation of the energetic azide $\alpha-\mathrm{NaN}_{3}^{\dagger}$}

\author{
Adam A. L. Michalchuk, (D) ab Svemir Rudić, (D) Colin R. Pulham (D) *ab and \\ Carole A. Morrison iD *a
}

\begin{abstract}
As initiation of an energetic material requires rupture of a covalent bond, and therefore population of antibonding electronic states, consideration of the electronic band gap has dominated initiation mechanisms for solid state materials. Most prominent are models based on metallisation, where static mechanical perturbation leads to closing of the electronic band gap. This work explores an alternative mechanism for the dynamic metallisation of a model energetic material, where vibrational excitation resulting from mechanical impact is found to induce transient metallisation of $\alpha-\mathrm{NaN}_{3}$. The normal coordinates associated with bending the azido anion close the electronic band gap, facilitating the formation of highly reactive species important for initiation of energetic materials. The DFT simulated vibrational spectrum of $\alpha-\mathrm{NaN}_{3}$ exhibits excellent reproduction of the experimental low-temperature inelastic neutron scattering spectrum (INS).
\end{abstract}

\section{Introduction}

Amongst the most important characteristics of an energetic material (EM, explosives, propellants and pyrotechnics) is its propensity to initiate on mechanical or thermal perturbation. At present it is necessary to first synthesise a candidate EM and then experimentally probe its sensitivity. Without a priori knowledge of EM sensitivity, the preparation of these materials is associated with considerable risk to safety. It is therefore of paramount importance to develop a theoretical framework capable of rationalising and predicting the impact sensitivity of EMs. In addition, this framework is crucial to the selective and targeted design of new materials.

The rapid release of energy that occurs following the initiation of an energetic material is the result of covalent bond rupture, typically $\mathrm{C}-\mathrm{N}, \mathrm{N}-\mathrm{N}$ or $\mathrm{N}-\mathrm{O}$. From a chemical perspective, logic dictates that molecules that contain weaker energetic bonds should display a higher propensity to initiate on perturbation. Indeed, the correlation of bond dissociation energies with sensitivity has been promising in many cases. ${ }^{1}$ However, physical models based purely on the dissociation of an isolated gasphase molecule are incapable of rationalising the notably different sensitivities displayed by e.g. different polymorphs or

\footnotetext{
${ }^{a}$ EaStCHEM School of Chemistry and Centre for Science at Extreme Conditions, University of Edinburgh, Edinburgh, UK. E-mail: c.r.pulham@ed.ac.uk, carole.morrison@ed.ac.uk

${ }^{b}$ EPSRC Centre for Continuous Manufacturing and Crystallisation (CMAC), Edinburgh, UK

'ISIS Neutron and Muon Source, STFC, Rutherford Appleton Laboratory, Chilton, Didcot, UK

$\dagger$ Electronic supplementary information (ESI) available. See DOI: 10.1039/c8cp06161k
}

multicomponent materials that contain the same energetic molecule. ${ }^{2-4}$ A variety of other correlations have been explored, including the study of electrostatic potentials, ${ }^{50}$ and both empirical and semi-empirical parameterisation..$^{1,5-7}$

As many energetic materials exist in the solid state, it has been suggested that initiation models should also be based on the solid state. While various correlations have been made, including non-covalent interaction lengths ${ }^{8}$ and void space, ${ }^{9}$ it is most common to rely on the study of electronic band gaps of EMs. ${ }^{10}$ These models can be rationalised by noting that bond dissociation requires population of anti-bonding states. Hence electronic excitation generally leads to a weakening of interatomic interactions. Materials that exhibit smaller band gaps can reach an excited electronic state more easily and hence are more prone to weakening of the energetic bond. This methodology is increasingly widespread, being dubbed as the 'band gap criterion' for energetic sensitivity. ${ }^{11}$ Moreover, a number of investigations have suggested considerable change to the band gap and dissociation energies under statically stressed solids. ${ }^{12-14}$ Another model has recently been developed, introducing the concept of pressure-induced metallisation of energetic materials. ${ }^{15}$ It was found that the pressures required for metallisation (i.e. electronic band gap narrowing to zero) typically fall between 20-100 GPa. These pressures are well above those responsible for structural phase transitions in these types of materials, and may indicate the importance of considering high-pressure phases when studying initiation and detonation phenomena. For example, high pressure studies have shown $\alpha$-FOX-7 to undergo a structural phase transition at ca. $2.5 \mathrm{GPa}(300 \mathrm{~K})$, with a second transition occurring near $5.7 \mathrm{GPa}(300 \mathrm{~K}) .{ }^{16,17} \mathrm{~A}$ number of pressure-induced phase 
transitions have also been observed in $\mathrm{RDX},{ }^{18} \mathrm{HMX},{ }^{19} \mathrm{CL}-20,{ }^{20}$ FOX-7, ${ }^{17,21}$ and a variety of other molecular energetic materials. The inorganic azides are also known to undergo structural phase transitions at ambient temperature on compression, for example $\beta-\mathrm{NaN}_{3} \rightarrow \alpha-\mathrm{NaN}_{3} \rightarrow \gamma-\mathrm{NaN}_{3} \rightarrow \delta-\mathrm{NaN}_{3}$ observed at 0.5, 14.0 and $27.6 \mathrm{GPa}$, respectively. ${ }^{22,23}$

An alternative picture of impact-induced reactions was recently proposed for the inorganic azides, ${ }^{24}$ with similar models having proven successful in molecular materials. ${ }^{25}$ In this model, it is suggested that the impact sensitivity of EMs rests in understanding the up-pumping of vibrational energy. ${ }^{26}$ The input energy that results from a mechanical perturbation is initially deposited into the lowest frequency vibrational states of the solid-state material, i.e. the lattice or phonon modes. These highly excited vibrational states subsequently relax, and 'pump' the energy upwards towards localised molecular vibrations. If the correct vibrational modes are excited, a non-adiabatic vibronic process may permit electronic excitation by reducing the band gap between the ground and excited state. Bond dissociation can then be considered on an excited state potential surface. Thus, these models encompass all aspects of previous models from incorporation of impact energy, to the mechanically-induced electronic excitation and bond rupture.

Central to the ab initio calculation of vibrational up-pumping is an understanding of the vibrational energy landscape. Inelastic neutron scattering spectroscopy (INS) has been shown to offer a powerful means against which to validate computational modelling of vibrational structure. These data have been used to validate a broad range of systems, including a number of molecular energetic materials. ${ }^{17,27}$ However, to date, no such validation has been performed for an energetic azide.

Following from a description of the vibrational structure, the up-pumping model requires identification of the so-called target frequency, $\omega_{\mathrm{T}}{ }^{26}$ That is, the molecular normal coordinate responsible for the initiation of the explosophoric species. In the previous work, the identification of $\omega_{\mathrm{T}}$ for the azide materials was based on a study of an isolated azido anion species in the gas phase. ${ }^{24}$ While this offered a clear and rapid identification of $\omega_{\mathrm{T}}$, it offers little by means of justification within a crystalline material. The present manuscript thus aims to explore whether the nature of $\omega_{\mathrm{T}}$ identified for a gas-phase molecule is justified once placed within a crystalline environment. This work also seeks to explore whether the structural distortions induced by the crystalline eigenvectors offer a route to induce transient metallisation, and hence provide an initiation mechanism for $\mathrm{NaN}_{3}$. Importantly, this mechanism differs from the conventional metallisation pathway, in that the stress induced by an eigenvector (and thus the existence of the metallised state) is transient, rather than static or continuous, as induced by an external pressure or shear strain.

\section{Methods}

Inelastic neutron scattering spectroscopy (INS)

INS spectra were collected on the TOSCA instrument ${ }^{28,29}$ at the ISIS Neutron and Muon Source. A powder sample of $\mathrm{NaN}_{3}$
(Aldrich $>99.5 \%$, ca $1.5 \mathrm{~g}, \alpha-\mathrm{NaN}_{3}$ at $T<c a .17 \mathrm{~K}^{30}$ ) was loaded into an aluminium sachet and sealed in a flat thinwalled aluminium sample holder. The sample was cooled to $<10 \mathrm{~K}$ and data collected to a total of $415 \mu \mathrm{A} \mathrm{h}$ (for approx. 3 hours). Data were processed using the Mantid software, ${ }^{31}$ and simulated spectra generated using AbINS. ${ }^{32}$

\section{Condensed matter calculations}

Input unit cell geometry was taken from the experimentally determined structure, deposited in the Inorganic Crystal Structural Database (ICSD, FIZ Karlsruhe, Code $34676 ;^{33}$ ca. $200 \mathrm{~K}$ ). Geometry optimisation was performed using plane wave Density Functional Theory (pw-DFT) as implemented in CASTEP v16. ${ }^{34}$ The GGA functional of Perdew-Burke-Ernzerhof $(\mathrm{PBE})^{35}$ was used, with Grimme's D2 dispersion correction, ${ }^{36}$ i.e. PBE-D2. The unit cell was fully relaxed to ensure minimization of all forces below $5 \times 10^{-4} \mathrm{eV}^{-1}$, with the electronic structure expanded in plane waves up to a kinetic energy cut-off of $1800 \mathrm{eV}$ and sampled on a Monkhorst-Pack ${ }^{37} k$-point grid with spacing $0.03 \AA^{-1}$. Norm-conserving pseudopotentials were used throughout. The optimised structural parameters are given in Table 1 . Zone-centre ( $\Gamma$-point) vibrational modes were calculated using the linear response method, with the acoustic sum rule applied in reciprocal space. LO-TO splitting was not included at $k=0$. The dynamical matrix was subsequently calculated across a set of 14 evenly-spaced $k$-points and interpolated over a fine grid.

Electronic band structures were modelled based on the same optimised geometry used for the phonon calculations. The equilibrium geometry was perturbed according to the zonecentre phonon eigenvectors. These perturbed structures were used as input for electronic band structure calculations. Further band structures were generated in CRYSTAL $17^{38}$ using the HSE06 ${ }^{39}$ hybrid DFT functional, which has been demonstrated to offer closer agreement with experimental band gaps for a broad range of materials compared to GGA functionals. ${ }^{24,40}$ Those presented here can therefore be regarded as accurate to within a reasonable level of confidence. Note also that as all simulations were performed as closed shell, all interpretations of the electronic band structure excitations refer to singlet channels only (that is, the spin forbidden intersystem crossing channels are not defined).

Table 1 Comparison of the optimised unit cell parameters for the primitive $(P)$ and conventional $(C)$ cells, using the D2 and TS dispersion corrections. The experimental X-ray structures (ca. $200 \mathrm{~K}$ ) are taken from ref. 33

\begin{tabular}{lllllll}
\hline & $a$ & $b$ & $c$ & $\alpha$ & $\beta$ & $\gamma$ \\
\hline$P^{\exp }$ & 3.608 & 3.608 & 5.413 & 105.360 & 105.360 & 60.963 \\
$P^{\text {cal,D2 }}$ & 3.589 & 3.589 & 5.198 & 101.818 & 101.818 & 61.768 \\
$P^{\text {cal,TS }}$ & 3.610 & 3.610 & 5.149 & 103.219 & 103.219 & 60.938 \\
$C^{\exp }$ & 6.211 & 3.658 & 5.323 & 90 & 107.225 & 90 \\
$C^{\text {cal,D2 }}$ & 6.161 & 3.685 & 5.198 & 90 & 103.806 & 90 \\
$V\left(P^{\mathrm{D} 2}\right) / V(\exp )$ & & & & & \\
$V\left(\mathrm{P}^{\mathrm{TS}}\right) / V(\exp )$ & & $-1.1 \%$ & & & \\
$V\left(\mathrm{C}^{\mathrm{D} 2}\right) / V(\exp )$ & & $-2.2 \%$ & & &
\end{tabular}




\section{Results and discussion}

\section{Primitive cell $-\alpha-\mathrm{NaN}_{3}$}

The primitive cell setting of $\alpha-\mathrm{NaN}_{3}$ contains only a single azido anion, and thus conforms to the chemical composition of the formula unit. The $\mathrm{N}_{3}{ }^{-}$anion (point group $D_{\infty \mathrm{h}}$ ) sits on a crystallographic site with site symmetry $C_{2 \mathrm{~h}}$. The vibrational symmetry of the molecule therefore breaks according to

$$
\begin{gathered}
\Pi_{\mathrm{u}} \rightarrow\left\{\begin{array}{c}
\mathrm{A}_{\mathrm{u}} \\
\mathrm{B}_{\mathrm{u}}
\end{array}\right. \\
\Sigma_{\mathrm{g}} \rightarrow \mathrm{A}_{\mathrm{g}} \\
\Sigma_{\mathrm{u}} \rightarrow \mathrm{A}_{\mathrm{u}}
\end{gathered}
$$

There are a total of nine optical modes in the first Brillouin zone of the primitive cell, with $\Gamma_{\text {optical }}=2 \mathrm{~A}_{\mathrm{g}}+2 \mathrm{~A}_{\mathrm{u}}+1 \mathrm{~B}_{\mathrm{g}}+4 \mathrm{~B}_{\mathrm{u}}$, which manifest as the four internal (molecular) and five external (lattice) modes listed in Table 2.

To verify the calculated vibrational frequencies, the inelastic neutron scattering spectrum (INS) of powdered $\alpha-\mathrm{NaN}_{3}$ was measured. This was compared with simulated INS spectra, based on the zone centre vibrational modes of the primitive cell, Fig. 1. The INS spectrum suggests that the lowest frequency modes should appear at approximately 130, 160, 196, 210 and $220 \mathrm{~cm}^{-1}$. The lowest frequency modes are Raman active, and agree with previously reported Raman spectra obtained at $100 \mathrm{~K}^{41}$ The calculations based on PBE-D2 appear to overestimate the lowest two vibrational modes by $15-20 \mathrm{~cm}^{-1}$, although the higher frequency lattice modes are very well reproduced, differing by no more than $c a .5 \mathrm{~cm}^{-1}$. As the lowest modes are highly dependent on the underlying potential, the zone-centre frequencies were recalculated using the dispersion correction of Tkatchenko and Scheffler (TS). ${ }^{42}$ Whilst this led to a

Table 2 Calculated zone-centre ( $\Gamma$-point) vibrational frequencies for the primitive and conventional unit cells of $\alpha-\mathrm{NaN}_{3}$ using PBE-D2

\begin{tabular}{llrl}
\hline Mode & $\omega$ primitive & $\omega$ conventional & Assignment \\
\hline $\mathbf{M}_{4}$ & - & 84.78 & Lattice \\
$\mathbf{M}_{5}$ & - & 88.61 & Lattice \\
$\mathbf{M}_{6}$ & 150.97 & 151.41 & Lattice \\
$\mathbf{M}_{7}$ & - & 154.47 & Lattice \\
$\mathbf{M}_{8}$ & - & 157.09 & Lattice \\
$\mathbf{M}_{9}$ & 178.00 & 184.34 & Lattice \\
$\mathbf{M}_{10}$ & 202.21 & 206.42 & Lattice \\
$\mathbf{M}_{11}$ & 214.51 & 219.05 & Lattice \\
$\mathbf{M}_{12}$ & 220.22 & 221.37 & Lattice \\
$\mathbf{M}_{13}$ & - & 221.54 & Lattice \\
$\mathbf{M}_{14}$ & - & 226.97 & Lattice \\
$\mathbf{M}_{15}$ & - & 230.22 & Lattice \\
$\mathbf{M}_{16}$ & - & 236.87 & Lattice \\
$\mathbf{M}_{17}$ & 606.28 & 610.35 & In phase $\delta \theta_{\mathrm{NNN}}$ \\
$\mathbf{M}_{18}$ & 609.72 & 614.72 & In phase $\delta \theta_{\mathrm{NNN}}$ \\
$\mathbf{M}_{19}$ & - & 617.49 & Out of phase $\delta \theta_{\mathrm{NNN}}$ \\
$\mathbf{M}_{20}$ & - & 618.24 & Out of phase $\delta \theta_{\mathrm{NNN}}$ \\
$\mathbf{M}_{21}$ & - & 1247.99 & Out of phase $\delta R_{\mathrm{S}}$ \\
$\mathbf{M}_{22}$ & 1250.43 & 1250.25 & In phase $\delta R_{\mathrm{S}}$ \\
$\mathbf{M}_{23}$ & - & 1929.65 & Out of phase $\delta R_{\mathrm{A}}$ \\
$\mathbf{M}_{24}$ & 1959.81 & 1964.65 & In phase $\delta R_{\mathrm{A}}$ \\
& & & \\
& & &
\end{tabular}

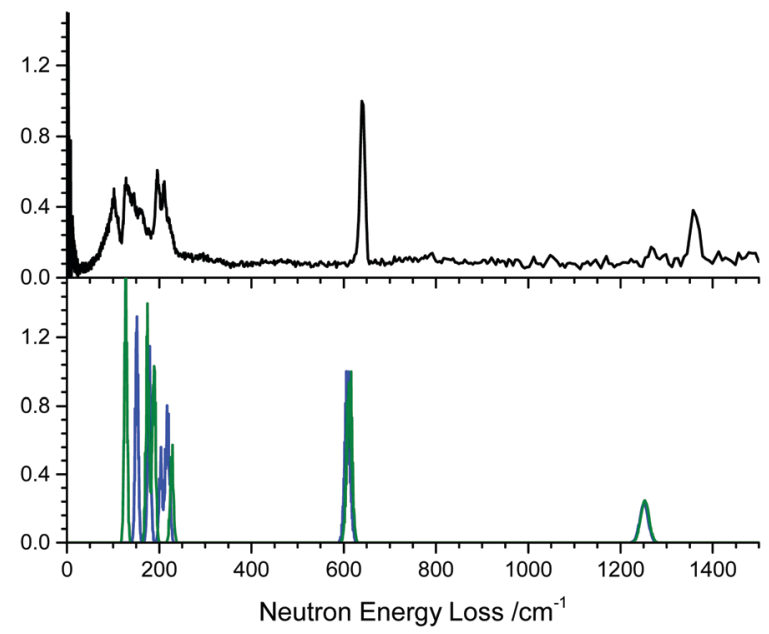

Fig. 1 Inelastic neutron spectrum for $\alpha-\mathrm{NaN}_{3}$. The experimental spectrum (top, recorded at $10 \mathrm{~K}$ ) is compared with the calculated spectra (bottom) based on zone-centre calculations using the D2 (blue) and TS (green) dispersion corrections.

slight improvement for the lowest frequency modes (at 127 and $174 \mathrm{~cm}^{-1}$ ), it led to poorer agreement for the higher frequency lattice modes (at $187 \mathrm{~cm}^{-1}, 189 \mathrm{~cm}^{-1}$ and $226 \mathrm{~cm}^{-1}$ ), ESI, $\dagger$ Section 1.

Note there is an additional broad feature present in the INS spectrum from $c a .80-100 \mathrm{~cm}^{-1}$ which is not reproduced in any of the calculated spectra or in literature Raman spectra. Consideration of the full phonon dispersion curves for primitive $\alpha-\mathrm{NaN}_{3}$ reveals the presence of van Hove singularities (i.e. kinks) in the acoustic branches about this frequency, Fig. 2A. This feature can therefore be ascribed to scattering from an acoustic mode away from the Brillouin zone centre and provides initial validation for the calculated dispersion curves. Interestingly, the intensity of this scattering at $c a .100 \mathrm{~cm}^{-1}$ is greater at lower momentum transfer, $Q$ (ESI, $\dagger$ Section 2), which suggests complex coherence of this feature and will make for an intriguing follow-up investigation. Simulation of the INS spectrum based on the full phonon dispersion calculation leads to substantially improved reproduction of the experimental spectrum, Fig. 2B, improving significantly as the sampling of the calculated phonon dispersion curve is increased. The experimentally observed intensities become more appropriately reproduced, the broadening is well matched, and the low frequency features around $100 \mathrm{~cm}^{-1}$ are now captured. This offers validation of the general phonon dispersion structure that is produced by DFT-based calculations for this system and highlights the importance of a complete understanding of the vibrational spectrum for modelling lattice structure in systems that display high $Q$-dependence of $\omega$.

The next vibrational band observed in the experimental spectrum is the $\mathrm{N}_{3}{ }^{-}$bend at $640 \mathrm{~cm}^{-1}$, which is $c a .30 \mathrm{~cm}^{-1}$ higher than predicted computationally. This $<5 \%$ underestimation is consistent with expected performance for pw-DFT(PBE) vibrational frequency calculations, including those reported previously for this material. ${ }^{22}$ The next mode 


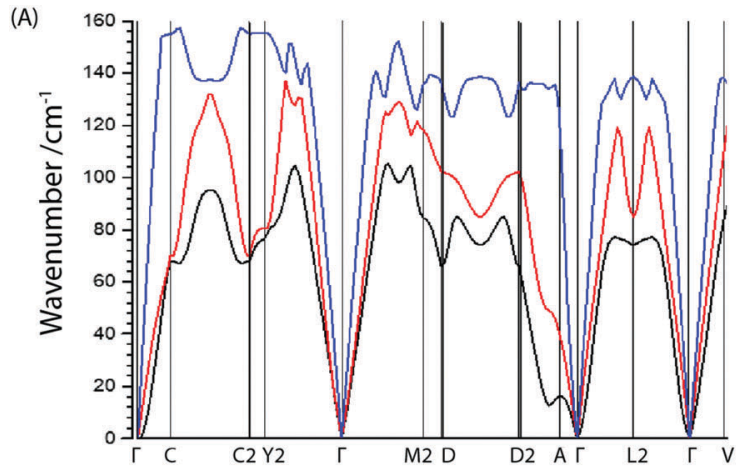

(B)

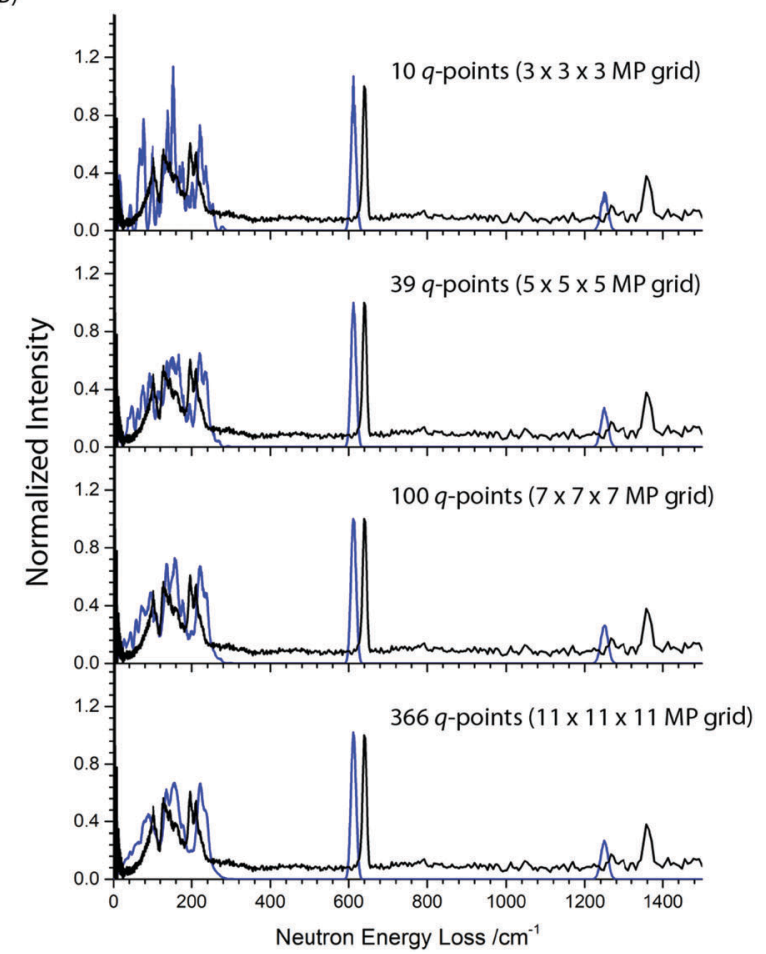

Fig. 2 (A) Simulated phonon dispersion curve for the three acoustic branches in primitive $\alpha-\mathrm{NaN}_{3}$. (B) The effect of increasing phonon grid size (blue curves) on the simulation of the INS spectrum for $\alpha-\mathrm{NaN}_{3}$.

is the symmetric stretch which at $1370 \mathrm{~cm}^{-1}$ in the INS spectrum is also underestimated by the PBE-D2 calculation, but this time by as much as $100 \mathrm{~cm}^{-1}$. The INS frequency is consistent with literature Raman measurements, ${ }^{43}$ suggesting the mis-match is due to limitations in the computational model. Previous attempts at calculating zone-centre frequencies with pw-DFT(PBE) also report underestimating this mode by the same amount. ${ }^{44}$ While the reason for the disagreement is unclear, we can conclude that that the model used in this study represents the experimental structure as well as could be expected. The asymmetric stretching mode (calculated at $1959 \mathrm{~cm}^{-1}$ without LO-TO and $2037 \mathrm{~cm}^{-1}$ with LO-TO) does not appear in the INS spectrum. This peak absence is attributed to a negligible incoherent scattering cross-section for ${ }^{14} \mathrm{~N}$ combining with a low vibrational amplitude. However, Raman spectroscopy has suggested this mode occurs around
$2040 \mathrm{~cm}^{-1},{ }^{43}$ and hence our calculations underestimate this mode by less than $1 \%$.

\section{Conventional cell of $\alpha-\mathrm{NaN}_{3}$}

The conventional unit cell of $\alpha-\mathrm{NaN}_{3}$ is the result of doubling the primitive unit cell contents, and thus comprises two azido anions and two sodium cations. By doubling the cell size, and hence halving the size of the Brillouin zone, a total of 24 vibrational modes ( 8 internal and 16 external) can be expected. These 24 modes correspond to the set of 12 modes that are the previously discussed zone-centre vibrational modes, and a further set of 12 modes in which the eigenvectors of each of the neighbouring primitive cells are directly out of phase with each other. A comparison of the calculated vibrational frequencies for the primitive and conventional cell is given in Table 2 .

In order to consider the effect of molecular perturbations on the electronic structure of the crystalline solid, we have chosen to impose perturbations of the normal coordinates on the conventional unit cell. This permits study of the edge of the first Brillouin zone, where the motions of the atoms in the two neighbouring primitive unit cells are out of phase, as well as the centre of the Brillouin zone, where the motions of the atoms are in phase, commensurate with the eigenvectors as expressed within the primitive unit cell.

For the purpose of the following discussion, it is convenient to define a perturbative term associated with 'walking' along each eigenvector, such that

$$
U_{i}=\alpha \varepsilon_{i} \mathbf{R}_{\text {eqm }}
$$

where $\varepsilon$ describes the mass reduced eigenvector of mode $i$ that perturbs the equilibrium atomic position $\mathbf{R}_{\text {eqm }}$ by a factor of $\alpha$. It is convenient to begin discussion of external vibrational modes that contain no Na character, with calculated frequencies of 151.41, 157.09, 184.34 and $236.87 \mathrm{~cm}^{-1}$ in the conventional cell-based calculation (i.e. $\mathrm{M}_{6}, \mathrm{M}_{8}, \mathrm{M}_{9}$ and $\mathrm{M}_{16}$ ). These four modes correspond to tilting of the azido anion molecules (as shown in Fig. 3). In the first, azide molecules tilt in phase, polarized primarily along the crystallographic $b$-axis. The second corresponds to a tilt along this same axis, with each of the azido anions tilting out of phase with one another. Hence, these modes correspond to the zone centre and Brillouin zone edge, respectively. $\mathrm{M}_{9}$ and $\mathrm{M}_{16}$ describe the same motion, but polarized primarily along the crystallographic $a$-axis.

Considering first the tilting modes along the $b$-axis $\left(\mathrm{M}_{6}\right.$ and $\mathrm{M}_{8}$ ), Fig. $3 \mathrm{~A}$ and $\mathrm{B}$, it was found that an enormous reduction in the band gap is observed if either of the two eigenvectors are followed. However, this appears to be an artefact of the rectilinear nature of the imposed eigenvectors. Indeed, if the $\mathrm{N}-\mathrm{N}$ bond lengths are corrected to their equilibrium values, this trend towards metallisation is lost. Only a small reduction in the band gap is observed at very large perturbations from the equilibrium geometry. However, the large difference observed between rectilinear and corrected distortions clearly shows that the addition of internal molecular modes may be a promising mechanism to induce transient metallisation.

The normal mode $\mathrm{M}_{9}$ is akin to the zone centre azide tilt along the crystallographic $a$-axis. If the associated eigenvector 

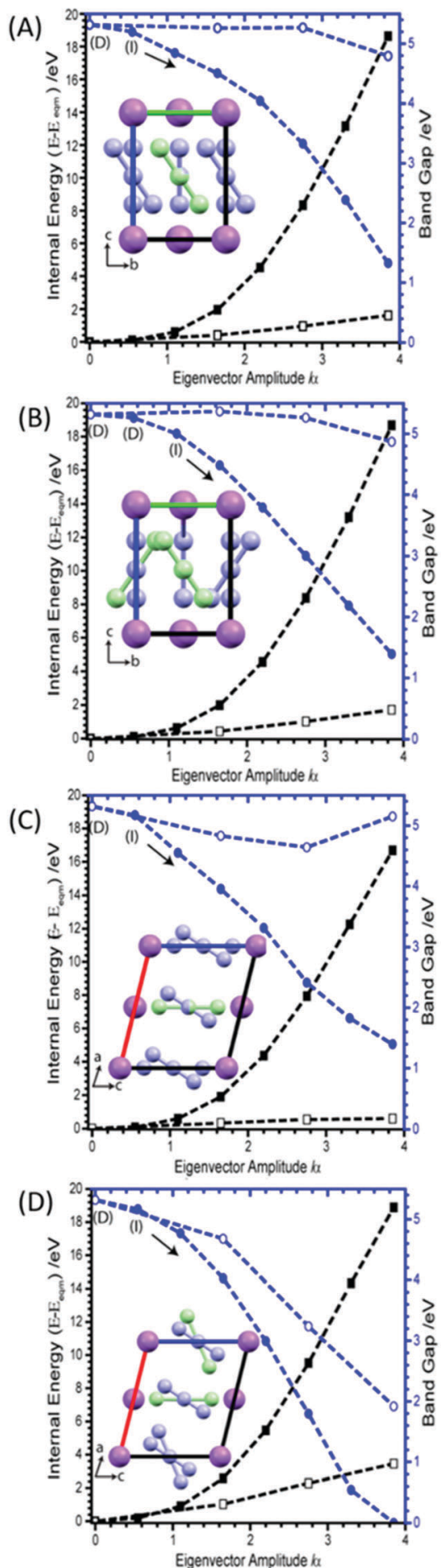

Fig. 3 Tilting of the azido anion molecule along the crystallographic $b$-axis, showing (A) $M_{6}$ (B) $M_{8}$, (C) $M_{9}$ and (D) $M_{16}$. Closed symbols are derived from rectilinear application of eigenvectors, and open symbols result after correcting $\mathrm{N}-\mathrm{N}$ bond lengths to equilibrium values. Internal (black) and band gap (blue) energies plotted on left and right $Y$-axes, respectively. The band gap is initially a direct band gap (D) but becomes indirect on perturbation (I). Inset graphics show the distortion (green molecule) with respect to the unperturbed (purple) molecular position. is followed, the band gap is seen to decrease very slightly before returning towards its equilibrium band gap at higher distortions, Fig. 3C. In stark contrast, the eigenvector of $\mathbf{M}_{16}$ is associated with a considerable decrease in the band gap, Fig. 3D. Analysis of the canonical orbitals suggests that this results from overlap of the out-of-phase $\pi$ non-bonding orbitals of the two $\mathrm{N}_{3}{ }^{-}$anions. Imposing large perturbations along this eigenvector (to a factor of $\alpha=8$ ) decreases the band gap further to $c a .0 .15 \mathrm{eV}$ (with increased internal energy of $c a .5 .5 \mathrm{eV}$ ), suggesting potential metallization when vibrational modes at the edge of the Brillouin zone are considered (ESI, $\dagger$ Section 3).

Interestingly, however, analysis of the band structure shows that this metallisation does not result from a closing of the azido anion band gap, but rather a reduction in the $\mathrm{N} \cdots \mathrm{Na}$ band gap, Fig. 4A. This can be found to result largely from an increase in energy of the $\mathrm{N}$ valence bands by approximately $3.5 \mathrm{eV}$. Metallisation in this manner suggests oxidation of the azido anion may occur under extreme excitations of the lattice modes in $\alpha-\mathrm{NaN}_{3}$, leading to transient formation of the neutral $\mathrm{N}_{3}$ radical. This is particularly interesting given previous suggestions ${ }^{45}$ that azide decomposition occurs via

$$
\begin{gathered}
2 \mathrm{~N}_{3}{ }^{-}+\mathrm{M}^{+} \rightarrow 2 \mathrm{~N}_{3}^{\bullet}+\mathrm{M}^{0} \\
2 \mathrm{~N}_{3}^{\bullet} \rightarrow 3 \mathrm{~N}_{2}
\end{gathered}
$$

(A)
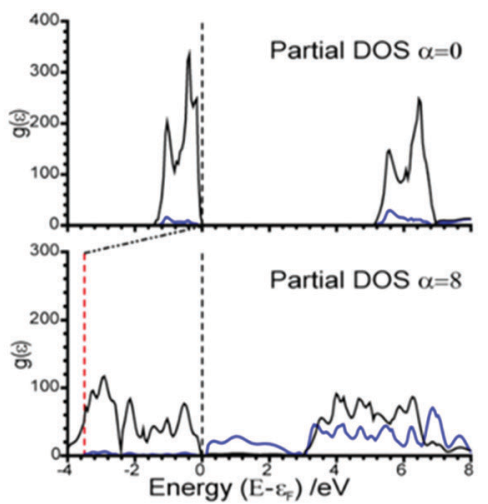

(B)
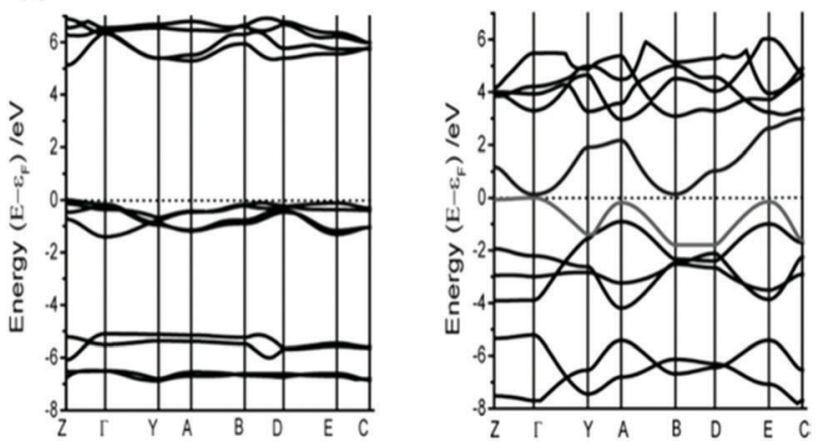

Fig. 4 Comparison of the electronic band gap at equilibrium geometry and after imposing an $\alpha=8$ fold perturbation of $M_{16}$. (A) The partial DOS is given for (black) $\mathrm{N}$ and (blue) Na species. The vertical dotted line on (A) is given to indicate the Fermi surface $\left(\varepsilon_{\mathrm{F}}\right)$, and the red vertical line shows the energy of $\varepsilon_{F}$ relative to the unperturbed structure. (B) The electronic band structure plotted along high symmetry lines in the Brillouin zone for (left) unperturbed and (right) $\alpha=8$ structures. 
Mechanical perturbation of a solid leads to large excitation of phonon modes. Because the phonon heat capacity is much lower than the total heat capacity of the material, this same input energy leads to lower populations of the internal vibrational modes upon equilibration. ${ }^{26}$ Consequently, displacement along the external mode eigenvectors exceeds that of the internal modes, and hence metallisation via $\mathbf{M}_{16}$ can be expected to occur more readily than by internal modes. However, $\mathrm{NaN}_{3}$ is well known to be insensitive to initiation by impact. ${ }^{46}$ Thus, despite the ease with which $\mathbf{M}_{16}$ can induce metallisation, we suggest that this mode is unlikely to be responsible for decomposition of $\mathrm{NaN}_{3}$ by dynamic impact initiation. However, it may be important for equilibrium decomposition via a thermal mechanism. Analysis of the band structure shown in Fig. 4B shows that while the eigenvector for $\mathbf{M}_{16}$ does result in an indirect band gap, the minimum energy gap spans a very narrow range of $k$-space, and hence results in very few excitation channels. Excitation across an indirect band gap is known to be very slow. ${ }^{47}$ Thus as the impact-induced vibrational energy transfer processes are expected to occur on the sub-nanosecond timescale (picosecond for phonon-phonon dissipation), it is reasonable to suggest that, while metallisation may occur, electronic excitation along eigenvector $\mathbf{M}_{16}$ is simply too slow to be considered here as a route to detonation. Further work on the explicit consideration of electron dynamics is however required.

The remaining nine external modes all exhibit a mixture of $\mathrm{N}$ and $\mathrm{Na}$ displacement (see eigenvectors in ESI, $\dagger$ Section 4). It is reassuring to find that none of these modes lead to any notable decrease in the band gap, Fig. 5. There is typically no more than ca. $1 \mathrm{eV}$ decrease in band gap along any of these eigenvectors, with $\mathrm{M}_{12}$ (the out of phase translation of $\mathrm{Na}$ and $\mathrm{N}_{3}{ }^{-}$species along the crystallographic $c$-axis), actually leading to an overall increase in band gap. The only exception is $\mathbf{M}_{15}$, which at large distortions appears to reduce the band gap quite drastically. However, it must be noted that this is associated with an increase in energy of around $60 \mathrm{eV}$, due to the manifestation of short contacts between $\mathrm{Na}$ atoms for this eigenvector.

\section{Internal vibrational modes}

The next four vibrational modes $\left(\mathbf{M}_{17}-\mathbf{M}_{20}\right)$ correspond to the internal bending motion of the azido anions. $\mathbf{M}_{17}$ and $\mathbf{M}_{18}$ are the zone-centre (i.e. in phase) bending of the azido anions perpendicular to and along the crystallographic $b$-axis, respectively. $\mathbf{M}_{19}$ and $\mathbf{M}_{20}$ are the corresponding out of phase bending modes.

For the purpose of following the effects of $\mathbf{M}_{17}-\mathbf{M}_{20}$ on the electronic band gap, the rectilinear perturbation was applied according to eqn (1). In doing so, however, artificial elongation of $\mathrm{N}-\mathrm{N}$ bond lengths was introduced. To ensure that only the isolated angle bend normal coordinate was investigated the $\mathrm{N}-\mathrm{N}$ bond lengths were therefore restored to equilibrium geometries. Further, to offer a comparison to previous gasphase studies, ${ }^{24}$ and to represent the energy per primitive cell, the internal energy is normalised by the number of azido anions in the conventional cell.

As the azido anion was perturbed along the bending mode, $\theta_{\mathrm{NNN}}$, the band gap was found to steadily decrease, with the
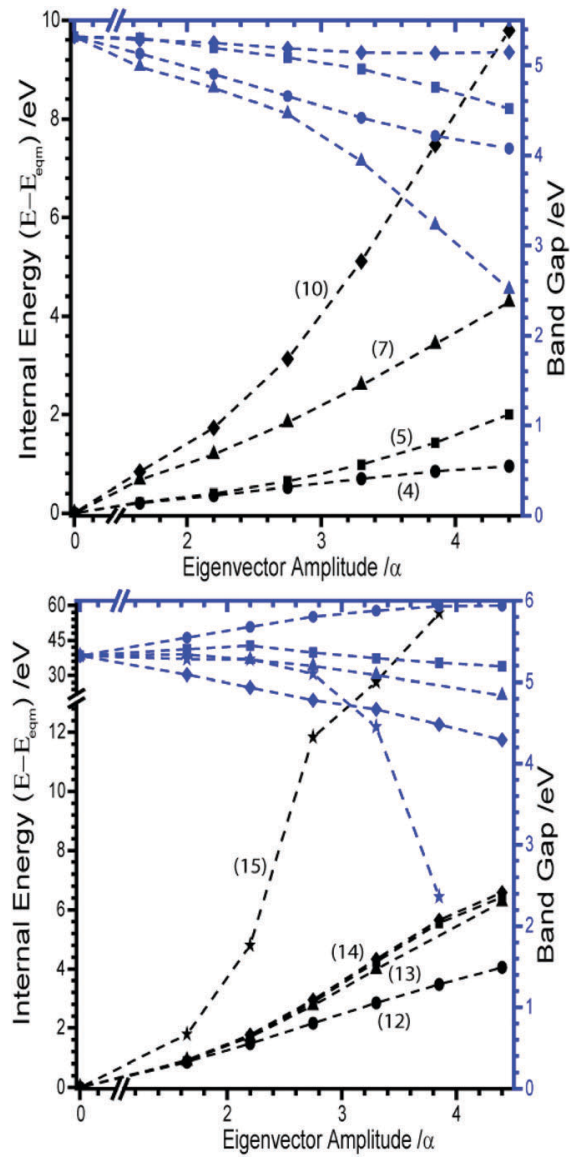

Fig. 5 Effect of external vibration normal coordinates on the energies and band gap of $\alpha-\mathrm{NaN}_{3}$. Internal (black) and band gap (blue) energies plotted on left and right $Y$-axes, respectively. Modes are identified as, (Top) $M_{4}(--), M_{5}(-\mathbf{-}-), M_{7}(-\boldsymbol{\Delta}-), M_{10}(-\boldsymbol{-})$, (bottom) $M_{11}(-\mathbf{-}-), M_{12}$ (- - ), $M_{13}(-\mathbf{\Delta}-), M_{14}(-\bullet-), M_{15}(-\star-)$.

band gap reaching approximately half of its original value at $\theta_{\mathrm{NNN}} \approx 130^{\circ}$. By $110^{\circ}$ the band gap drops to $0 \mathrm{eV}$, Fig. 6 .

Unlike for $\mathbf{M}_{16}$, the narrowing in the band gap that is observed on imposing normal coordinate perturbations 17-20 are not the result of $\mathrm{N} \cdots \mathrm{Na}$ band gap closing. Instead, metallization occurs by a closing of the $\mathrm{N}_{3}{ }^{-}$conduction/valence band gap, Fig. 7. The full band structure diagram also suggests that, in contrast to $\mathbf{M}_{16}$, very little increase in band dispersion occurs as metallization approaches. Thus whilst the band gap does switch from direct to indirect, the shortest crossing points persist across broad ranges of $k$-space, indicating very many more excitation channels exist across the Brillouin zone, in contrast to $\mathrm{M}_{16}$.

Note the behavior of the azide anion in the crystal lattice contrasts markedly with what has been reported for the same ion in the gas phase, ${ }^{24}$ where molecular perturbations of the vibrational eigenvectors could only locate conical intersections between $\mathrm{S}_{0} / \mathrm{T}_{1}$ electronic states, i.e. spin 'forbidden' channels. Thus the periodicity of the crystalline lattice has led not only to a notable (and expected) reduction in the molecular HOMOLUMO gap but has also opened up more probable singlet channels for electronic excitation. 
(A)

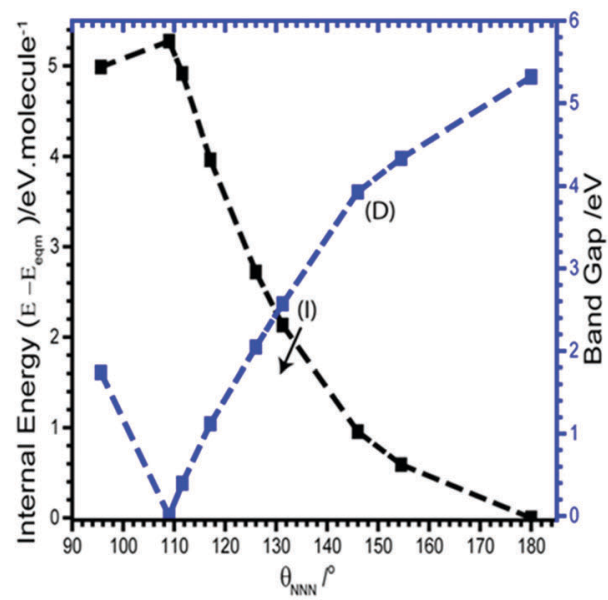

(B)

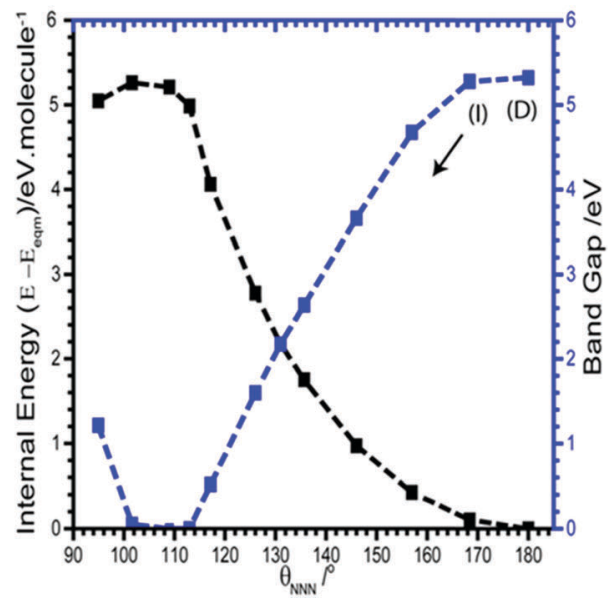

Fig. 6 Effect of (A) $M_{17}$ and (B) $M_{19}$ (B) on the band gaps and energies of $\alpha-\mathrm{NaN}_{3}$. Band gap momentum conservation is indicated as direct (D) or indirect (I). Internal (black) and band gap (blue) energies plotted on left and right $Y$-axes, respectively. The arrow indicates continuation of an indirect band gap. $M_{18}$ and $M_{20}$ are given in ESI, $\uparrow$ Section 5.

Within the confines of symmetry, metallisation permits electron transfer from the valence bands of the metal cation to the azido anion, leading to potential, albeit chemically unlikely, transient formation of $\mathrm{N}_{3}{ }^{2-}$. The ability to lower the conduction band to such considerable degrees also offers pathways to the role of local electron defects within these structures. Such processes have previously been suggested as being crucial to the initiation of energetic compounds, although no mechanism for their athermal influence was proposed. ${ }^{48,49}$ It therefore appears that coupling normal mode behavior with electronic excitation offers a route to explaining the formation of highly unstable species in energetic materials during the detonation process.

$\mathbf{M}_{21}$ is the out of phase symmetric stretching mode. Due to the contraction of one set of $\mathrm{N}-\mathrm{N}$ bonds over this normal coordinate it was not possible to follow this mode beyond a $\mathrm{N}-\mathrm{N}$ stretch of $1.4 \AA$. By this limit, the band gap was found to decrease only slightly, from $c a .5 .2$ to $3.3 \mathrm{eV}$. It is therefore unlikely that this mode provides a route to metallization. The in-phase (zone centre) symmetric stretching mode is
(A)

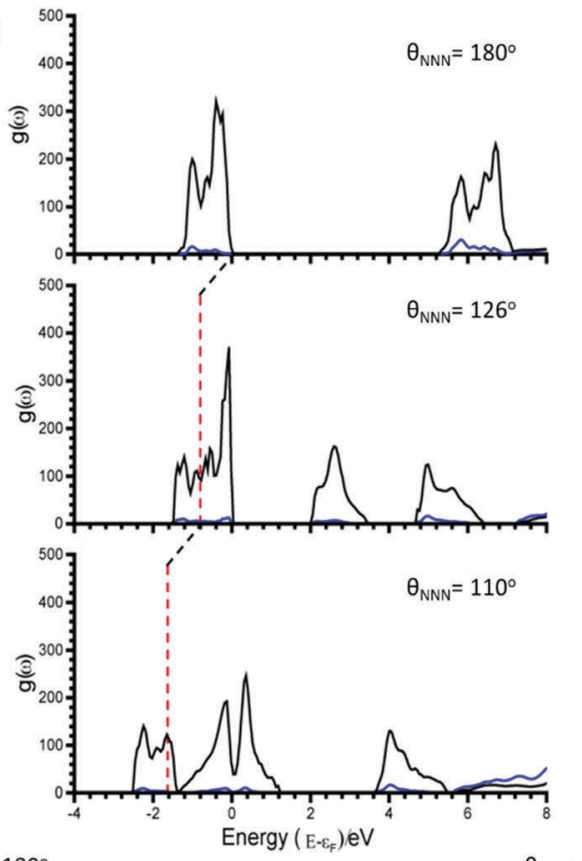

(B)

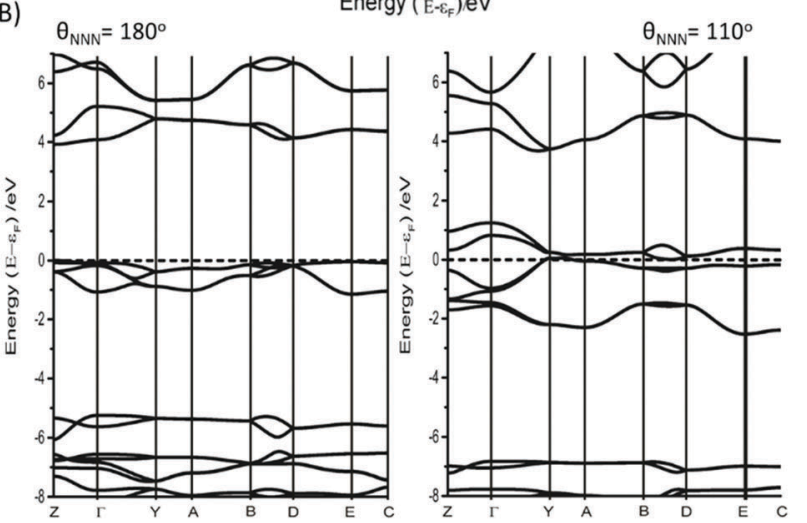

Fig. 7 (A) Partial electronic density of states as a function of $M_{17}$, with contributions from $\mathrm{N}_{3}^{-}$(black) and $\mathrm{Na}^{+}$(blue). The relative energy of $\varepsilon_{\mathrm{F}}$ at $\theta_{\mathrm{NNN}}=180^{\circ}$ is shown as a red vertical line in the perturbed structures. (B) Electronic band structures for $\mathrm{NaN}_{3}$ with $\theta_{\mathrm{NNN}}=180^{\circ}$ and $\theta_{\mathrm{NNN}}=110^{\circ}$. $\varepsilon_{\mathrm{F}}$ is marked with a dotted line.

expressed as $\mathbf{M}_{22}$. By the same change in internal energy $\left(\right.$ ca. $+5 \mathrm{eV}$ molecule $\left.{ }^{-1}\right)$ at which the bending modes led to metallisation, the band gap from the symmetric stretching mode had reduced to only $c a$. $2 \mathrm{eV}$. Further extension of this normal coordinate unsurprisingly leads to metallisation as the $\mathrm{N}-\mathrm{N}$ bonds are ruptured. However, this occurs with an increase of internal energy $>12 \mathrm{eV}$ molecule ${ }^{-1}$ (Fig. 8A). The final two normal coordinates, $\mathbf{M}_{23}$ and $\mathrm{M}_{24}$, are the in- and out-of-phase asymmetric stretching modes, respectively and behave in the same manner. For the same reason described for $\mathbf{M}_{21}$, there is a physical limitation on the maximum $U_{i}$ that can be applied to these modes. This limit was reached with an energy penalty of $c a .13 \mathrm{eV}$ molecule ${ }^{-1}$, by which point the band gap was found to reduce to only $3.8 \mathrm{eV}$ (Fig. 8B). Thus, metallisation can only be attained via the bending normal coordinate, consistent with findings for the gas phase $\mathrm{N}_{3}{ }^{-}$ molecule. 
(A)

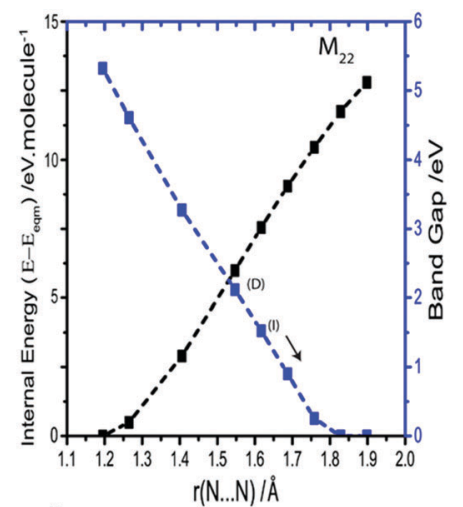

(B)

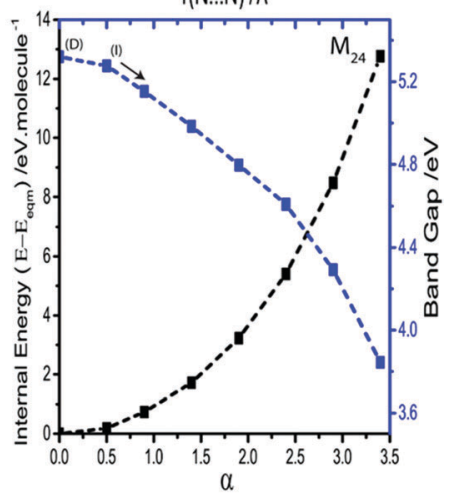

Fig. 8 Effect of $M_{22}$ and $M_{24}$ (i.e. the symmetric and asymmetric stretches, respectively) on the electronic band gap. The latter is plotted as $\alpha$, defined as $r=\left(r_{\text {eqm }}+\alpha / 10\right)$, where $r_{\text {eqm }}$ is the equilibrium bond length. Band gaps are defined as direct (D) or indirect (I) and the arrow indicates that the indirect band gap continues. To reflect perturbation of two azido anions (in the conventional cell), energy is given per molecule.

\section{Conclusions}

Impact sensitivity is a crucial parameter in understanding the performance, safety and application of energetic materials. To date, little has been understood concerning the physical parameters that govern the sensitivity of these materials. It has recently been demonstrated that a vibrational up-pumping model offers a powerful base upon which to understand this phenomenon. Within this model, the azide materials were suggested to initiate via the bending normal coordinate of the $\mathrm{N}_{3}{ }^{-}$species.

The present manuscript demonstrates the ability of density functional theory to faithfully reproduce most components of the vibrational spectrum of the well-known inorganic azide $\alpha \mathrm{NaN}_{3}$. This was done by comparison with low temperature experimental inelastic neutron scattering spectra. The high levels of vibrational dispersion exhibited by this crystalline system required complete modelling of vibrations across the Brillouin zone in order to fully reproduce the INS spectrum. As the normal coordinates were followed away from the equilibrium structure, the electronic band gap was calculated for the conventional unit cell. For the thirteen external optical modes, only one was found to induce an indirect band gap reduction (between $\mathrm{N}$ and $\mathrm{Na}$ bands) to give viable excitation channels but over a restricted range of $k$-space. Whilst excitation across an indirect band gap may be expected to be slow, it is possible that this crossing may offer a mechanism for formation of radical $N_{3}$ in the solid. However as $\mathrm{NaN}_{3}$ is well known to be insensitive to impact initiation it is unlikely that the detonation model is dependent on a readily excitable low energy phonon mode. A more thorough understanding of why this external mode does not lead to initiation is therefore required.

Consideration of the internal modes clearly demonstrates that vibrationally-induced, transient metallisation of the azido anion within the solid state can be readily achieved through the bending mode eigenvector. Narrowing the angle in the azide anion by about $60^{\circ}$, at the cost of around $5 \mathrm{eV}$ per molecular unit, results in a closing of the valence and conduction band. This suggests that an $\mathrm{S}_{0} / \mathrm{S}_{1}$ conical intersection may be available in the solid state which is absent in the gas phase. The electronic band gap narrowing may account for the formation of the highly reactive $\mathrm{N}_{3}{ }^{2-}$ species, which has been suggested previously to be crucial in the initiation of energetic compounds. The up-pumping model for impact induced initiation of energetic materials therefore holds when the effect of the crystalline environment is considered. We suggest that the concept of vibrationally induced metallisation, resulting in transient band gap narrowing, can lead to the creation of reactive species in crystalline solids, and may be key to understanding reactivity under the highly vibrationally excited states that are associated with shocked materials.

\section{Conflicts of interest}

There are no conflicts to declare.

\section{Acknowledgements}

INS Spectra were collected on the TOSCA instrument at the ISIS Neutron and Muon Facility (RB 1710382). The authors thank EPSRC CMAC EP/I033459/1 (AM) for funding. Thanks to the UK Materials and Molecular Modelling Hub for computational resources, partially funded by EPSRC (EP/P020194/1). Additional thanks to the Edinburgh Compute and Data Facilities (ECDF) for further computational resources. Final thanks to Prof Adam Cumming and Dr Stewart Parker for many helpful discussions.

\section{Notes and references}

1 D. Mathieu and T. Alaime, J. Phys. Chem. A, 2014, 118, 9720-9726.

2 B. W. Asay, B. F. Henson, L. B. Smilowitz and P. M. Dickson, J. Energ. Mater., 2003, 21, 223-235.

3 K. B. Landenberger, O. Bolton and A. J. Matzger, J. Am. Chem. Soc., 2015, 137, 5074-5079.

4 S. R. Kennedy and C. R. Pulham, in Co-crystals: Preparation, Characterization and Applications, ed. C. B. Aakeröy and A. S. Sinha, Royal Society of Chemistry, Cambridge, UK, 2018, pp. 231-266. 
5 D. Mathieu, Ind. Eng. Chem. Res., 2017, 56, 8191-8201.

6 N. R. Badders, C. Wei, A. A. Aldeeb, W. J. Rogers and M. S. Mannan, J. Energ. Mater., 2006, 24, 17-33.

7 R. V. Tsyshevsky, O. Sharia and M. M. Kuklja, Molecules, 2016, 21, 236.

8 M. Cartwright and J. Wilkinson, Propellants, Explos., Pyrotech., 2010, 35, 326-332.

9 P. Politzer and J. S. Murray, J. Mol. Model., 2014, 20, 2233.

10 W. Zhu, J. Xiao and H. Xiao, J. Phys. Chem. B, 2006, 110, 9856-9862.

11 H. Zhang, F. Cheung, F. Zhao and X. Cheng, Int. J. Quantum Chem., 2009, 109, 1547-1552.

12 M. M. Kuklja, S. N. Rashkeev and F. J. Zerilli, Appl. Phys. Lett., 2006, 89, 071904.

13 M. M. Kuklja and S. N. Rashkeev, Appl. Phys. Lett., 2007, 90, 2005-2008.

14 M. M. Kuklja and S. N. Rashkeev, Appl. Phys. Lett., 2007, 90, 151913.

15 S. V. Bondarchuk, J. Phys. Chem. A, 2018, 122, 5455-5463.

16 M. M. Bishop, N. Velisavljevic, R. Chellappa and Y. K. Vohra, J. Phys. Chem. A, 2015, 119, 9739-9747.

17 S. Hunter, P. L. Coster, A. J. Davidson, D. I. A. Millar, S. F. Parker, W. G. Marshall, R. I. Smith, C. A. Morrison and C. R. Pulham, J. Phys. Chem. C, 2015, 119, 2322-2334.

18 I. D. H. Oswald, D. I. A. Millar, A. J. Davidson, D. J. Francis, W. G. Marshall, C. R. Pulham, A. Cumming, A. R. Lennie and J. E. Warren, High Pressure Research, 2010, vol. 30, pp. 280-291.

19 F. Goetz, T. Brill and J. Ferraro, J. Phys. Chem., 1978, 82, 1912-1917.

20 J. Li and T. B. Brill, Propellants, Explos., Pyrotech., 2007, 32, 326-330.

21 Z. A. Dreger, Y. Tao and Y. M. Gupta, Chem. Phys. Lett., 2013, 584, 83-87.

22 D. Li, P. Zhu, J. Jiang, D. Xu, R. Liu, X. Wang, Q. Cui and H. Zhu, J. Phys. Commun., 2017, 1, 025002.

23 D. I. A. Millar, C. Barry, W. G. Marshall and C. R. Pulham, Z. Kristallogr., 2014, 229, 259-275.

24 A. A. L. Michalchuk, P. T. Fincham, P. Portius, C. R. Pulham and C. A. Morrison, J. Phys. Chem. C, 2018, 122, 19395-19408.

25 J. Bernstein, J. Chem. Phys., 2018, 148, 084502.

26 D. D. Dlott and M. D. Fayer, J. Chem. Phys., 1990, 92, 3798-3812.

27 S. Hunter, T. Sutinen, S. F. Parker, C. A. Morrison, D. M. Williamson, S. Thompson, P. J. Gould and C. R. Pulham, J. Phys. Chem. C, 2013, 117, 8062-8071.

28 S. F. Parker, F. Fernandez-Alonso, A. J. Ramirez-Cuesta, J. Tomkinson, S. Rudic, R. S. Pinna, G. Gorini and J. Fernández Castañon, J. Phys. Conf. Ser., 2014, 554, 012003.

29 R. S. Pinna, S. Rudić, S. F. Parker, J. Armstrong, M. Zanetti, G. Škoro, S. P. Waller, D. Zacek, C. A. Smith, M. J. Capstick,
D. J. McPhail, D. E. Pooley, G. D. Howells, G. Gorini and F. Fernandez-Alonso, Nucl. Instrum. Methods Phys. Res., Sect. A, 2018, 896, 68-74.

30 S. R. Aghdaee and A. I. M. Rae, Acta Crystallogr., Sect. B: Struct. Sci., 1984, 40, 214-218.

31 O. Arnold, J. C. Bilheux, J. M. Borreguero, A. Buts, S. I. Campbell, L. Chapon, M. Doucet, N. Draper, R. Ferraz Leal, M. A. Gigg, V. E. Lynch, A. Markvardsen, D. J. Mikkelson, R. L. Mikkelson, R. Miller, K. Palmen, P. Parker, G. Passos, T. G. Perring, P. F. Peterson, S. Ren, M. A. Reuter, A. T. Savici, J. W. Taylor, R. J. Taylor, R. Tolchenov, W. Zhou and J. Zikovsky, Nucl. Instrum. Methods Phys. Res., Sect. A, 2014, 764, 156-166.

32 K. Dymkowski, S. F. Parker, F. Fernandez-Alonso and S. Mukhopadhyay, Phys. B, 2018, 1-6, in Press.

33 G. E. Pringle and D. E. Noakes, Acta Crystallogr., Sect. B: Struct. Crystallogr. Cryst. Chem., 1968, 24, 262-269.

34 S. J. Clark, M. D. Segall, C. J. Pickard, P. J. Hasnip, M. I. J. Probert, K. Refson and M. C. Payne, Z. Kristallogr., 2005, 220, 567-570.

35 J. P. Perdew, K. Burke and M. Ernzerhof, Phys. Rev. Lett., 1996, 77, 3865-3868.

36 S. Grimme, J. Comput. Chem., 2006, 27, 1787-1799.

37 J. D. Pack and H. J. Monkhorst, Phys. Rev. B: Condens. Matter Mater. Phys., 1976, 13, 5188-5192.

38 R. Dovesi, R. Orlando, A. Erba, C. M. Zicovich-Wilson, B. Civalleri, S. Casassa, L. Maschio, M. Ferrabone, M. De La Pierre, P. D’Arco, Y. Noël, M. Causà, M. Rérat and B. Kirtman, Int. J. Quantum Chem., 2014, 114, 1287-1317.

39 A. V. Krukau, O. A. Vydrov, A. F. Izmaylov and G. E. Scuseria, J. Chem. Phys., 2006, 125, 224106.

40 A. J. Garza and G. E. Scuseria, J. Phys. Chem. Lett., 2016, 7, 5-10.

41 Z. Iqbal, J. Chem. Phys., 1973, 59, 1769-1774.

42 A. Tkatchenko and M. Scheffler, Phys. Rev. Lett., 2009, 102, 6-9.

43 J. I. Bryant, J. Chem. Phys., 1964, 40, 3195-3203.

44 K. Ramesh Babu, C. Bheema Lingam, S. P. Tewari and G. Vaitheeswaran, J. Phys. Chem. A, 2011, 115, 4521-4529.

45 W. Zhu, X. Xu and H. Xiao, J. Phys. Chem. Solids, 2007, 68, 1762-1769.

46 H. D. Fair and R. F. Walker, Energetic Materials 1: Physics and Chemistry of the Inoganic Azides, Plenum Press, New York, 1977.

47 T. Kirchartz and U. Rau, J. Phys. Chem. Lett., 2017, 8, 1265-1271. 48 B. P. Aduev, E. D. Aluker, V. G. Kriger and Y. A. Zakharov, Solid State Ionics, 1997, 101-103, 33-36.

49 B. P. Aduev, É. D. Aluker, G. M. Belokurov, A. N. Drobchik, Y. A. Zakharov, A. G. Krechetov and A. Y. Mitrofanov, Combust., Explos. Shock Waves, 2000, 36, 622-632.

50 P. Politzer and J. Murray, J. Mol. Struct., 1996, 376, 419-424. 